\title{
GENERAL ALGORITHM AND SENSITIVITY ANALYSIS FOR VARIATIONAL INEQUALITIES ${ }^{1}$
}

\author{
MUHAMMAD ASLAM NOOR \\ King Saud University \\ Mathematics Department, College of Science \\ P.O. Box 2455 \\ Riyadh 11451 \\ Saudi Arabia
}

\begin{abstract}
The fixed point technique is used to prove the existence of a solution for a class of variational inequalities related to odd order boundary value problems, and to suggest a general algorithm. We also study the sensitivity analysis for these variational inequalities and complementarity problems using the projection technique. Several special cases are discussed, which can be obtained from our results.

Key words: Variational inequalities, Complementarity problems, Sensitivity analysis, Fixed point method, Projection method, Odd order boundary value problems.
\end{abstract}

AMS (MOS) subject classifications: 49A29, 49A10, 90C30, $90 \mathrm{C} 25,65 \mathrm{~K} 10,47 \mathrm{~K} 10$.

\section{INTRODUCTION}

Variational inequality theory is a very useful and effective technique for studying a wide class of problems in a unified natural and general framework. This theory has been extended and generalized in several directions using new and powerful methods that have led to the solution of basic and fundamental problems previously thought to be inaccessible. Some of these developments have made mutually enriching contacts with other areas of the mathematical and engineering sciences. In recent years, the author has considered and studied a new class of variational inequalities, which enabled him to study both odd and even order boundary value problems having constrained conditions. In this paper, we use the auxiliary variational principle technique to prove the existence of the solution of variational inequalities for odd order boundary value problems and suggest a general iterative algorithm. We also study the qualitative behavior of the solution of the variational inequalities when the given operator and the feasible convex set vary with a parameter. Such a study is known as

\footnotetext{
${ }^{1}$ Received: October, 1990. Revised: June, 1991.
} 
sensitivity analysis, which is also important and meaningful. Sensitivity analysis provides us useful information for designing various equilibrium systems, and for predicting the future changes of the equilibria as a result of the changes in the governing systems. In addition, from a theoretical point of view, sensitivity properties of a mathematical programming problem can provide new insight concerning the problems being studied and can sometime stimulate new ideas and techniques for solving them.

Motivated and inspired by the recent research work going on in this area, we consider a new class of variational inequalities. Using the fixed point technique of Glowinski, Lions and Tremolieres [3], and Noor [7,8], we prove the existence of a solution of this variational inequality. This approach enables us to suggest and analyze a general algorithm for these variational inequalities. We also show that the variational inequality problem is equivalent to solving a fixed point problem using the projection method. This equivalence is used to study the sensitivity analysis of the parametric variational inequality. This approach is due to Dafermos [2]. We also consider the sensitivity analysis for the general complementarity problems. Several special cases are also discussed.

In Section 2, we formulate the variational inequality problem and review some necessary basic results. The existence of the solution of variational inequality problem is studied in Section 3 using the fixed point method along with a general algorithm. Sensitivity analysis is the subject of Section 4 . The applications of the main results are considered in Section 5 .

\section{VARIATIONAL INEQUALITY FORMULATION}

Let $H$ be a real Hilbert space with norm and inner product denoted by $\|\cdot\|$ and $\langle\cdot, \cdot\rangle$ respectively. Let $K$ be a nonempty closed convex set in $H$.

Given $T, g: H \rightarrow H$ continuous operators, consider the functional $I[v]$, defined by

$$
I[v]=\frac{1}{2}\langle T v, g(v)\rangle
$$

which is known as the general energy (cost) functional. Note that for $g=I$, the identity operator, the functional $I[v]$, defined by $(2.1)$ becomes:

$$
I_{1}[v]=\frac{1}{2}\langle T v, v\rangle
$$

which is the classical energy functional.

If the operator $T$ is linear, $g$-symmetric, that is 


$$
\langle T(u), g(v)\rangle=\langle g(u), T(v)\rangle, \text { for all } u, v \in H,
$$

and $g$-positive definite, then one can show that the minimum of $I[v]$, defined by $(2.1)$ on the convex set $K$ in $H$ is equivalent to finding $u \in H$ such that $g(u) \in K$ and

$$
\langle T u, g(v)-g(u)\rangle \geq 0, \text { for all } g v) \in K .
$$

The inequality (2.2) is known as the general variational inequality, introduced and studied by Noor [9]. We remark that if $g=I$, the identity operator, then problem (2.2) is equivalent to finding $u \in K$ such that

$$
\langle T u, v-u\rangle \geq 0, \text { for all } v \in K,
$$

which is known as the variational inequality problem considered and studied by Lions and Stampacchia [6].

If $K=H$, then the problem (2.2) is equivalent to finding $u \in H$ such that

$$
\langle T u, g(v)\rangle=0, \text { for all } g(v) \in H .
$$

The problem (2.4) is known as the weak formulation of the odd order boundary value problems and this appears to be a new one.

To illustrate this fact, we consider the third order two-point boundary value problem

$$
\begin{gathered}
-D^{3} u(x)=f(x), \quad 0<x<1 \\
u(0)=u^{\prime}(0)=u^{\prime}(1)=0,
\end{gathered}
$$

where $f(x)$ is a given function of $x$. Now using the technique of $K$-positive definite operators, as developed in [12], we can show that the problem (2.5) is equivalent to finding $u \in H_{0}^{2}[0,1]$, a Hilbert space [6], such that

$$
\langle T u, K v\rangle=\langle f(x), K v\rangle, \text { for all } K v \in H_{0}^{2}[0,1]
$$

where

$$
\langle T u, K v\rangle=-\int_{0}^{1} D^{3} u D v d x=\int_{0}^{1} D^{2} u D^{2} v d x
$$

and

$$
\langle f(x), K v\rangle=\int_{0}^{1} f(x) K v d x
$$

with 


$$
K=D=\frac{d}{d x} .
$$

It is clear that with $g=K$, we have the weak formulation (2.4) of the third order boundary value problem (2.5).

If $K^{*}=\{u \in H ;\langle u, v\rangle \geq 0$ for all $v \in K\}$ is a polar cone of the convex cone $K$ in $H$ and $K \subset g(K)$, then problem (2.2) is equivalent to finding $u \in H$ such that

$$
g(u) \in K, T u \in K^{*} \text { and }\langle T u, g(u)\rangle=0 .
$$

The problem (2.6) is known as the general complementarity problem. Note the symmetry role played by the operators $T$ and $g$, since $K=K^{*}=R_{+}^{N}$. This problem includes many previously known problems as special cases.

It is clear that problems (2.3), (2.4) and (2.6) are special cases of the general variational inequality (2.2) introduced in this paper. In brief, we conclude that the problem (2.2) is a most general and unifying one, which is one of the main motivations of this paper.

Definition 2.1: We say that an operator $T: H \rightarrow H$ is said to be

(a) Strongly monotone, if there exists a constant $\alpha>0$ such that

$$
\langle T u-T v, u-v\rangle \geq \alpha\|u-v\|^{2}, \text { for all } u, v \in H
$$

(b) Lipschitz continuous, if there exists a constant $\beta>0$ such that

$$
\|T u-T v\| \leq \beta\|u-v\|, \text { for all } u, v \in H .
$$

It is clear that if $\beta$ exists, then so does $\alpha$, and $\alpha \leq \beta$.

\section{EXISTENCE THEORY}

In this section, using the fixed point technique of Glowinski, Lions and Tremolieres [3] and Noor $[7,8]$, we prove the existence of the solution of the general variational inequality (2.2).

Theorem 3.1: Let the operators $T, g: H \rightarrow H$ be both strongly monotone and Lipschitz continuous respectively. If the operator $g$ is one-to-one, then there exists a unique solution $u \in H$ such that $g(u) \in K$, a closed convex set in $H$ and

$$
\langle T u, g(v)-g(u)\rangle \geq 0, \text { for all } g(v) \in K
$$

Proof: 
Uniqueness: Its proof is similar to that of Glowinski, Lions and Tremolieres [3].

Existence: We now use the fixed point technique to prove the existence of the solution of (3.1). For given $u \in H$, we consider the auxiliary problem of finding $w \in H$ such that $g(w) \in K$ satisfying the variational inequality

$$
\langle w, v-w\rangle \geq\langle u, v-w\rangle-\rho\langle T u, g(v)-g(w)\rangle, \text { for all } g(v) \in K,
$$

where $\rho>0$ is a constant.

Let $w_{1}, w_{2}$ be two solutions of (3.2) related to $u_{1}, u_{2} \in H$ respectively. It is enough to show that the mapping $u \rightarrow w$ has a fixed point belonging to $H$ satisfying (3.1). In other words, we have to show that for $\rho>0$ well chosen

$$
\left\|w_{1}-w_{2}\right\| \leq \theta\left\|u_{1}-u_{2}\right\|
$$

with $0<\theta<1$, where $\theta$ is independent of $u_{1}$ and $u_{2}$. Taking $v=w_{2}$, (respectively $w_{1}$ ) in (3.2) related to $u_{1}$ (respectively $u_{2}$ ), we have

$$
\left\langle w_{1}, w_{2}-w_{1}\right\rangle \geq\left\langle u_{1}, w_{2}-w_{1}\right\rangle-\rho\left\langle T u_{1}, g\left(w_{2}\right)-g\left(w_{1}\right)\right\rangle
$$

and

$$
\left\langle w_{2}, w_{1}-w_{2}\right\rangle \geq\left\langle u_{2}, w_{1}-w_{2}\right\rangle-\rho\left\langle T u_{2}, g\left(w_{1}\right)-g\left(w_{2}\right)\right\rangle .
$$

Adding these inequalities, we obtain

$$
\begin{gathered}
\left\langle w_{1}-w_{2}, w_{1}-w_{2}\right\rangle \leq\left\langle u_{1}-u_{2}, w_{1}-w_{2}\right\rangle-\rho\left\langle T u_{1}-T u_{2}, g\left(w_{1}\right)-g\left(w_{2}\right)\right\rangle \\
=\left\langle u_{1}-u_{2}-\rho\left(T u_{1}-T u_{2}\right), w_{1}-w_{2}\right\rangle \\
+\rho\left\langle T u_{1}-T u_{2}, w_{1}-w_{2}-\left(g\left(w_{1}\right)-g\left(w_{2}\right)\right)\right\rangle
\end{gathered}
$$

from which it follows that

$$
\begin{gathered}
\left\|w_{1}-w_{2}\right\|^{2} \leq\left\|u_{1}-u_{2}-\rho\left(T u_{1}-T u_{2}\right)\right\|\left\|w_{1}-w_{2}\right\| \\
+\rho\left\|w_{1}-w_{2}-\left(g\left(w_{1}\right)-g\left(w_{2}\right)\right)\right\|\left\|T u_{1}-T u_{2}\right\|
\end{gathered}
$$

Since $T, g$ are both strongly monotone and Lipschitz continuous, so by using the technique of Noor [10], we have

$$
\left\|u_{1}-u_{2}-\rho\left(T u_{1}-T u_{2}\right)\right\|^{2} \leq\left(1-2 \alpha \rho+\beta^{2} \rho^{2}\right)\left\|u_{1}-u_{2}\right\|^{2}
$$

and

$$
\left\|w_{1}-w_{2}-\left(g\left(w_{1}\right)-g\left(w_{2}\right)\right)\right\|^{2} \leq\left(1-2 \sigma+\delta^{2}\right)\left\|w_{1}-w_{2}\right\|^{2}
$$


From (3.3), (3.4) and (3.5), we obtain, by using the Lipschitz continuity of T,

$$
\begin{gathered}
\left\|w_{1}-w_{2}\right\| \leq\left\{\beta \rho\left(\sqrt{1-2 \delta+\sigma^{2}}\right)+\sqrt{1-2 \alpha \rho+\beta^{2} \rho^{2}}\right\}\left\|u_{1}-u_{2}\right\| \\
=\{\rho k+t(\rho)\}\left\|u_{1}-u_{2}\right\|, \\
=\theta\left\|u_{1}-u_{2}\right\|,
\end{gathered}
$$

where $\theta=\rho k+t(\rho)$

$$
k=\beta\left(\sqrt{1-2 \delta+\sigma^{2}} \text { and } t(\rho)=\sqrt{1-2 \alpha \rho+\beta^{2} \rho^{2}} .\right.
$$

We now show that $\theta<1$. It is clear that $t(\rho)$ assumes its minimum value for $\bar{\rho}=\frac{\alpha}{\beta^{2}}$ with $t(\bar{\rho})=\sqrt{1-\frac{\alpha^{2}}{\beta^{2}}}$. For $\rho=\bar{\rho}, \rho k+t(\rho)<1$ implies that $\rho k<1$ and $k<\alpha$. Thus it follows that $\theta<1$ for all $\rho$ with

$$
\rho<2 \frac{\alpha-k}{\beta^{2}-k^{2}}, \rho<\frac{1}{k} \text { and } k<\alpha .
$$

Since $\theta<1$, so the mapping $u \rightarrow w$ defined by (3.2) has a fixed point, which is the solution of (3.1), the required result.

Remark 3.1: If $g=I$, the identity operator, then the problem (3.2) is equivalent to find $w \in H$, for a given $u \in H$ such that

$$
\langle w, v-w\rangle \geq\langle u, v-w\rangle-\rho\langle T u, v-w\rangle, \text { for all } v \in K
$$

and $\rho>0$. From the proof of Theorem 3.1, we see that $k=0$ and $\theta=\sqrt{1-2 \alpha+\beta^{2} \rho^{2}}<1$ for $0<\rho<\frac{2 \alpha}{\beta^{2}}$, so the mapping $u \rightarrow w$ defined by (3.6) has a fixed point, which is the solution of the variational inequality (2.3), studied by Lions and Stampacchia [6]. Consequently, we have a new proof of the variational inequality problem (2.3).

Remark 3.2: We note that the solution of the variational inequality problem (3.2) is equivalent to finding the minimum of the function $F(w)$ on $K$, where

$$
F(w)=1 / 2\langle w, w\rangle-\langle u, w\rangle+\rho\langle T u, g(w)\rangle
$$

Here $\rho>0$ is a constant. It turns out that this auxiliary problem is very useful in suggesting iterative algorithm for computing an approximate solution of the variational inequality (3.1). Based on these observations, we extend the ideas of Cohen [1] and Noor [7] to propose a more general algorithm: 
General Algorithm 3.1: For some $u \in K$, we introduce the following auxiliary problem

$$
\min _{w \in K} F[w]
$$

where

$$
F[w]=E(w)+\rho\langle T u, g(w)\rangle-\left\langle E^{\prime}(u), w\right\rangle .
$$

Here $\rho$ is a constant and $E$ is a convex differentiable functional.

It is clear that the solution $w$ of (3.8) can be characterized by the variational inequality

$$
\left\langle E^{\prime}(w), u-w\right\rangle \geq\left\langle E^{\prime}(u), v-w\right\rangle-\rho\langle T u, g(v)-g(w)\rangle, \text { for all } v \in K
$$

We note that if $w=u$, then clearly $w$ is a solution of (3.1). This fact suggests the following iterative algorithm.

(i) Choose the initial vector $w_{0}$.

(ii) At step $n$, solve the auxiliary problem (3.8) with $u=w_{n}$. Let $w_{n+1}$ denote the solution of this problem.

(iii) Calculate $\left\|w_{n+1}-w_{n}\right\|$. If $\left\|w_{n+1}-w_{n}\right\| \leq \epsilon$, for given $\epsilon>0$, stop, otherwise repeat $(i i)$.

It is obvious that if $E(w)=\langle w, w\rangle$, then the general auxiliary problem (3.8) is exactly the same as (3.7). Therefore, one may consider this algorithm as a generalization of the previous ideas of Noor [7]. We remark that the general algorithm 3.1 is quite general and flexible. By appropriate choice of the auxiliary problem, one may be able to select a suitable method to solve the variational inequality and related optimization problems. Since the auxiliary problem (3.8) is essentially a minimization problem, a large number of algorithms are available to solve it.

\section{SENSITIVITY ANALYSIS}

In this section, we study the sensitivity analysis for the variational inequality of type (3.1). This problem has attracted considerable attention recently. The methodologies suggested so far vary with the problem settings being studied. Sensitivity analysis for variational inequalities has been studied by Tobin [14], Kyparisis [4,5], Dafermos [2] and Qiu and Magnanti [13] using quite different techniques. We mainly follow the ideas and technique 
of Dafermos [2], as extended by Noor [11] for a class of quasi variational inequalities, which is based on the projection technique. This approach has strong geometric flavor. Using this technique, one usually proves the equivalence between the variational inequality problem and the fixed point problem. Consequently, this technique implies that continuity, Lipschitz continuity and differentiability of the perturbed solution depends upon the continuity, Lipschitz continuity and differentiability of the projection operator on the family of feasible convex sets.

We now consider the parametric variational inequality version of problem (3.1). To formulate the problem, let $M$ be an open subset of $H$ in which the parameter $\lambda$ takes values and assume that $\left\{K_{\lambda}: \lambda \in M\right\}$ is a family of closed convex subsets of $H$. The parametric general variational inequality problem is:

Find $u \in H$ such that $g(u) \in K_{\lambda}$ and

$$
\langle T(u, \lambda), g(v)-g(u)\rangle \geq 0, \text { for all } g(v) \in K_{\lambda},
$$

where $T(u, \lambda)$ is a given operator defined on the set of $(u, \lambda)$ with $\lambda \in M$ and takes values in $H$. We also assume that for some $\bar{\lambda} \in M$, the problem (4.1) admits a solution $\bar{u}$. We want to investigate those conditions under which, for each $\lambda$ in a neighborhood of $\bar{\lambda}$, the problem (4.1) has a unique solution $u(\lambda)$ near $\bar{u}$ and the function $u(\lambda)$ is continuous, Lipschitz continuous and differentiable. We assume that $X$ is the closure of a ball in $H$ centered at $\bar{u}$.

We also need the following concepts.

Definition 4.1: Let $T$ be an operator defined on $X \times M$. Then for all $\lambda \in M$ and $u$, $v \in X$, the operator $T$ is said to be

a) Locally strongly monotone, if there exists a constant $\alpha>0$ such that

$$
\langle T(u, \lambda)-T(v, \lambda), u-v\rangle \geq \alpha\|u-v\|^{2}
$$

and

b) Locally Lipschitz continuous, if there exists a constant $\beta>0$ such that

$$
\|T(u, \lambda)-T(v, \lambda)\|,=\beta\|u-v\| .
$$

In particular, it follows that $\alpha \leq \beta$.

We need the following results to prove the main result of this section. The first one is due to Noor [9]. 
Lemma 4.1 [9]. The function $u \in K_{\lambda}$ is a solution of the parametric variational inequality (4.1) if and only if $u$ is the fixed point of the map:

$$
F(u, \lambda)=u-g(u)+P_{K_{\lambda}}[g(u)-\rho T(u, \lambda)],
$$

for all $\lambda \in M$ and some $\rho>0$, where $P_{K_{\lambda}}$ is the projection of $H$ of the family of closed convex sets $K_{\lambda}$.

We here consider the case, when the solutions of the variational inequality (4.1) lie in the interior of $X$. For this purpose, we consider the map,

$$
F^{*}(u, \lambda)=u-g(u)+P_{K_{\lambda} \cap X}[g(u)-\rho T(u, \lambda)], \text { for all }(u, \lambda) \in X \times M .
$$

We have to show that the map $F^{*}(u, \lambda)$ has a fixed point, which by Lemma 4.1 is also a solution of the variational inequality (4.1). First of all, we prove that the map $F^{*}(u, \lambda)$ is a contraction map with respect to $u$, uniformly in $\lambda \in M$ by using the locally strongly monotonicity and Lipschitz continuity of the operator $T(u, \lambda)$ defined on $X \times M$.

Lemma 4.2: $\quad$ For all $u, v \in X$, and $\lambda \in M$, we have

$$
\left\|F^{*}(u, \lambda)-F^{*}(v, \lambda)\right\| \leq \theta\|u-v\|
$$

where $\theta=k+t(\rho)<1$ for $\left|\rho-\frac{\alpha}{\beta^{2}}\right|<\frac{\sqrt{\alpha^{2}-\beta^{2}\left(2 k-k^{2}\right)}}{\beta^{2}}, \alpha>\beta \sqrt{k(k-2)}$ and $k<1$.

Proof: $\quad$ For all $u, v \in X, \lambda \in M$, we have from (4.5),

$$
\begin{gathered}
\left\|F^{*}(u, \lambda)-F^{*}(v, \lambda)\right\| \leq\|u-v-(g(u)-g(v))\| \\
+\left\|P_{K_{\lambda} \cap X}[g(u)-\rho T(u, \lambda)]-P_{K_{\lambda} \cap X}[g(v)-\rho T(v, \lambda)]\right\| \\
\leq 2\|u-v-(g(u)-g(v))\|+\|u-v-\rho(T(u, \lambda)-T(v, \lambda))\|
\end{gathered}
$$

since the projection operator $P_{K_{\lambda} \cap X}$ is nonexpansive, see [3].

Now the operators $T(u, \lambda)$ and $g$ are both (locally) strongly monotone and Lipschitz continuous, so by the method of Noor [10],

$$
\|u-v-\rho(T(u, \lambda)-T(v, \lambda))\|^{2} \leq\left(1-2 \alpha \rho+\beta^{2} \rho^{2}\right)\|u-v\|^{2}
$$

and

$$
\|u-v-(g(u)-g(v))\|^{2} \leq\left(1-2 \delta+\sigma^{2}\right)\|u-v\|^{2} .
$$

From (4.6), (4.7) and (4.8), we obtain 


$$
\begin{gathered}
\left\|F^{*}(u, \lambda)-F^{*}(v, \lambda)\right\| \leq\left\{2\left(\sqrt{1-2 \delta+\sigma^{2}}\right)+\sqrt{1-2 \alpha \rho+\beta^{2} \rho^{2}}\right\}\|u-v\| \\
=(k+t(\rho))\|u-v\| \\
=\theta\|u-v\|,
\end{gathered}
$$

where $\theta=k+t(\rho)$

$$
k=2\left(\sqrt{1-2 \delta+\sigma^{2}}\right) \text {, and } t(\rho)=\sqrt{1-2 \alpha \rho+\beta^{2} \rho^{2}} .
$$

Now, using the technique of Noor [9], one can show that $\theta<1$ for

$$
\left|\rho-\frac{\alpha}{\beta^{2}}\right|<\frac{\sqrt{\alpha^{2}-\beta^{2}\left(2 k-k^{2}\right)}}{\beta^{2}}, \alpha>\beta \sqrt{k(k-2)}
$$

and $k<1$, from which it follows that the map $F^{*}(u, \lambda)$ defined by (4.5) is a contraction map, the required result.

Remark 4.1: $\quad$ From Lemma 4.2, we see that the map $F^{*}(u, \lambda)$ has a unique fixed point $u(\lambda)$, that is $u(\lambda)=F^{*}(u, \lambda)$. Also by assumption, the function $\bar{u}$, for $\lambda=\bar{\lambda}$ is a solution of the parametric variational inequality (4.1). Again using Lemma 4.2, we see that $\bar{u}$ is a fixed point of $F^{*}(u, \lambda)$ and it is also a fixed point of $F^{*}(u, \bar{\lambda})$. Consequently, we conclude that $u(\bar{\lambda})=\bar{u}=F^{*}(u(\bar{\lambda}, \bar{\lambda})$.

Using Lemma 4.2 and the technique of Dafermos [2], we prove the continuity of the solution $u(\lambda)$ of the variational inequality (4.1), which is the motivation of our next result.

Lemma 4.3: If the operators $T(\bar{u}, \lambda), g(\bar{u})$ and the map $\lambda \rightarrow P_{K_{\lambda} \cap X}[g(\bar{u})-$ $\rho T(\bar{u}, \bar{\lambda}]$ are continuous (or Lipschitz continuous), then the function $u(\lambda)$ satisfying $(4.1)$ is continuous (or Lipschitz continuous) at $\lambda=\bar{\lambda}$.

Proof: $\quad$ For $\lambda \in M$, using Lemma 4.2 and the triangle inequality, we have

$$
\begin{gathered}
\| u\left(\lambda-u(\bar{\lambda})\|=\| F^{*}(u(\lambda), \lambda)-F^{*}(u(\bar{\lambda}), \bar{\lambda}) \|\right. \\
\left.\leq\left\|F^{*}(u(\lambda), \lambda)-F^{*}(u(\bar{\lambda}), \lambda)\right\|+\| F^{*}(u(\bar{\lambda}), \lambda)-F^{*} u(\bar{\lambda}), \bar{\lambda}\right) \| \\
\leq \theta\|u(\lambda)-u(\bar{\lambda})\|+\left\|F^{*}(u(\bar{\lambda}), \lambda)-F^{*}(u(\bar{\lambda}), \bar{\lambda})\right\| .
\end{gathered}
$$

From (4.5) and the fact that the projection map is nonexpansive, we have

$$
\begin{gathered}
\left\|F^{*}(u(\bar{\lambda}), \lambda)-F^{*}(u(\bar{\lambda}), \bar{\lambda})\right\| \\
=\| P_{K_{\lambda} \cap X}[g(u(\bar{\lambda}))-\rho T(u(\bar{\lambda}), \lambda)]-P_{K_{\lambda} \cap X}[g(u(\bar{\lambda})-\rho T(u(\bar{\lambda}), \bar{\lambda})] \| \\
\leq \rho\|T(u(\bar{\lambda}), \lambda)-T(u(\bar{\lambda}), \bar{\lambda})\|+\| P_{K_{\lambda} \cap X}[g(u(\bar{\lambda}))-\rho T(u(\bar{\lambda}), \bar{\lambda})]
\end{gathered}
$$




$$
-P_{K_{\bar{\lambda}} \cap X}[g(u(\bar{\lambda}))-\rho T(u(\bar{\lambda}), \lambda)] \|
$$

Now from Remark 4.1, and combining (4.9) with (4.10), we get

$$
\begin{gathered}
\|u(\lambda)-\bar{u}\| \leq \frac{\rho}{1-\theta}\|T(\bar{u}, \lambda)-T(\bar{u}, \bar{\lambda})\|+\frac{1}{1-\theta} \| P_{K_{\lambda} \cap X}[g(\bar{u})-\rho T(\bar{u}, \bar{\lambda})] \\
-P_{K_{\bar{\lambda}} \cap X}[g(\bar{u})-\rho T(\bar{u}, \bar{\lambda})] \|,
\end{gathered}
$$

from which, the required result follows.

Lemma 4.4: If the assumptions of Lemma 4.3 hold, then there exists a neighborhood $N \subset M$ of $\bar{\lambda}$ such that for $\lambda \in N, u(\lambda)$ is the unique solution of the parametric variational inequality (4.1) in the interior of $X$.

Proof: Its proof is similar to that of Lemma 2.5 in [2].

We now state and prove the main result of this section.

Theorem 4.1: $\quad$ Let $\bar{u}$ be the solution of the parametric variational inequality (4.1) and $\lambda=\bar{\lambda}$ and $T(u, \lambda)$ be the locally strongly monotone Lipschitz continuous operator for all $u$, $v \in X$. If the operators $T(\bar{u}, \lambda), g(\bar{u})$ and the map $\lambda \rightarrow P_{K_{\lambda} \cap X}[g(\bar{u})-\rho T(\bar{u}, \bar{\lambda})]$ are continuous (or Lipschitz continuous) at $\lambda=\bar{\lambda}$, then there exists a neighborhood $N \subset M$ of $\bar{\lambda}$ such that for $\lambda \in N$, the parametric variational inequality (4.1) has a unique solution $u(\lambda)$ in the interior of $X, u(\bar{\lambda})=\bar{u}$ and $u(\lambda)$ is continuous (or Lipschitz continuous) at $\lambda=\bar{\lambda}$.

Proof: $\quad$ Its proof follows from Lemmas $4.2-4.4$ and the Remark 4.1.

Remark 4.2: The results obtained in this section can be extended, when the operators $T$ and $g$ both are allowed to vary with the parameter $\lambda$ along with the feasible convex sets. The variational inequality problem (4.1) becomes:

Find $u \in H$ such that $g(u) \in K_{\lambda}$ and

$$
\left\langle T(u, \lambda), g(v, \lambda)-g(u, \lambda) \geq 0 \text { for all } g(v) \in K_{\lambda}\right.
$$

which is equivalent to finding $u \in H$ such that

$$
F_{1}(u, \lambda)=P_{K_{\lambda}}[g(u, \lambda)-\rho T(u, \lambda)]
$$

for $\lambda \in M$ and $\rho>0$. This formulation allows us to obtain the similar results as in Theorem 4.1 . 
Remark 4.3: We note that for $g=I$, the identity operator, the parametric variational inequality $(4.1)$ reduces to:

Find $u \in K_{\lambda}$ such that

$$
\langle T(u, \lambda), v-u\rangle \geq 0, \text { for all } v \in K_{\lambda}
$$

the problem studied by Dafermos. Consequently our results are exactly the same as proved in [2]. We also remark that the function $u(\lambda)$ as defined in Theorem 4.1 is continuously differentiable on some neighborhood $N$ of $\bar{\lambda}$. For this, see Dafermos [2].

\section{GENERAL COMPLEMENTARITY PROBLEM}

In this section, we illustrate that the results obtained in the Sections 3 and 4 can be used to study the sensitivity analysis for the general complementarity problem. To be more precise, given $T, g: H \rightarrow H$, find $u \in H$ such that

$$
g(u) \in K, T u \in K^{*} \text { and }\langle g(u), T u\rangle=0
$$

where $K^{*}$ is the polar cone of the convex cone $K$ in $H$. Since the problems (5.1) and (3.1) are equivalent, so the results of Theorem 3.1 and Theorem 4.1 can be used to prove the existence of a solution and the sensitivity analysis of the problem (5.1).

\section{ACKNOWLEDGMENT}

The author is indebted to the referee for helpful comments and suggestions.

\section{REFERENCES}

[1] G. Cohen, "Auxiliary problem principle extended to variational inequalities", J. Opt. Theor. Appl. 59, (1988), pp. 325-333.

[2] S. Dafermos, "Sensitivity analysis in variational inequalities", Math. Opers. Res. 13, (1988), pp. 421-434.

[3] R. Glowinski, J. Lions, and R. Tremolieres, "Numerical Analysis of Variational Inequalities", North-Holland, Amsterdam (1981).

[4] J. Kyparisis, "Sensitivity analysis framework for variational inequalities", Math. Programming 38, (1987), pp. 203-213.

[5] J. Kyparisis, "Perturbed solutions of variational inequality problems over polyhedral sets”, J. Optim. Theor. Appl. 57, (1988), pp. 295-305. 
[6] J. Lions and G. Stampacchia, "Variational inequalities", Comm. Pure Appl. Math. 20, (1967), pp. 493-519.

[7] M.A. Noor, "General nonlinear variational inequalities", J. Math. Anal. Appl. 126, (1987), pp. $78-84$.

[8] M.A. Noor, "Variational inequalities related with a Signorini problem", C.R. Math. Rep. Acad. Sci. Canada 7, (1985), pp. 267-272.

[9] M.A. Noor, “General variational inequalities”, Appl. Math. Lett. 1, (1988), pp. 119-122.

[10] M.A. Noor, “An iterative scheme for a class of quasi variational inequalities", J. Math. Anal. Appl. 110, (1985), pp. 463-468.

[11] M.A. Noor, "Some classes of variational inequalities in Constantin Caratheodory", An International Tribute, edited by T. Rassias, World Scientific Publishing Co., Singapore, London (1990).

[12] W.V. Petryshyn, "Direct and iterative methods for the solution of linear operator equations in Hilbert space”, Trans. Amer. Math. Soc. 105, (1962), pp. 136-175.

[13] Y. Qiu and T.L. Magnanti, "Sensitivity analysis for variational inequalities defined on polyhedral sets", Math. Oper. Res. 14, (1989), pp. 410-432.

[14] R.L. Tobin, "Sensitivity analysis for variational inequalities", J. Optim. Theor. Appl. 48, (1986), pp. 191-204. 


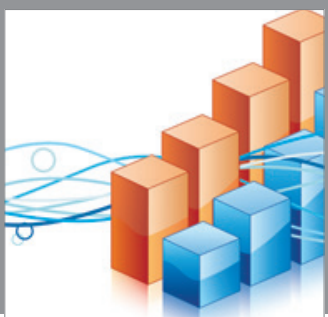

Advances in

Operations Research

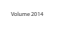

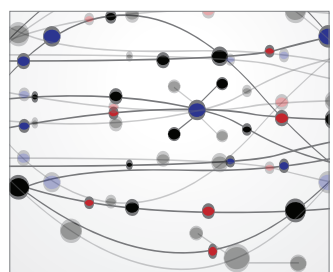

\section{The Scientific} World Journal
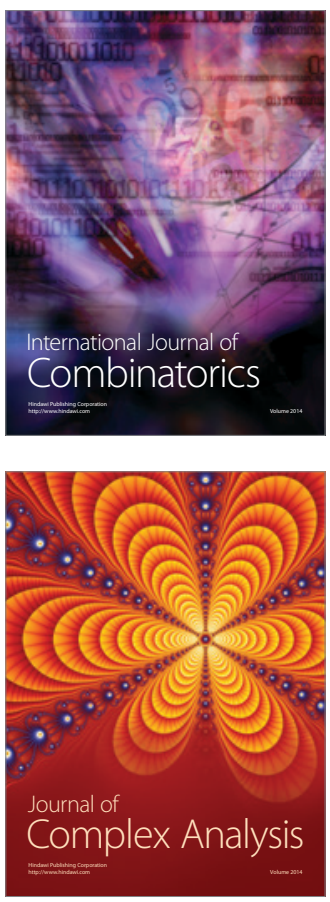

International Journal of

Mathematics and

Mathematical

Sciences
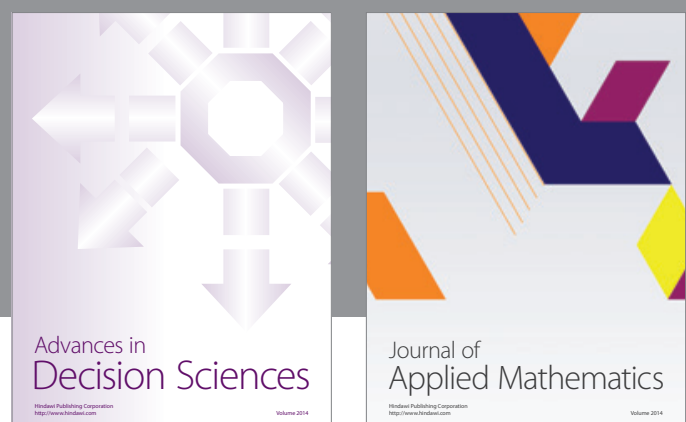

Journal of

Applied Mathematics
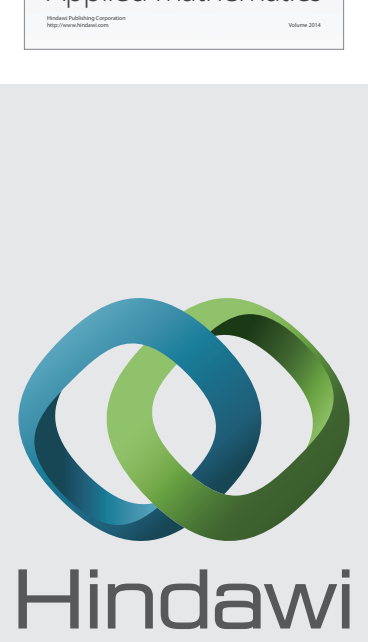

Submit your manuscripts at http://www.hindawi.com
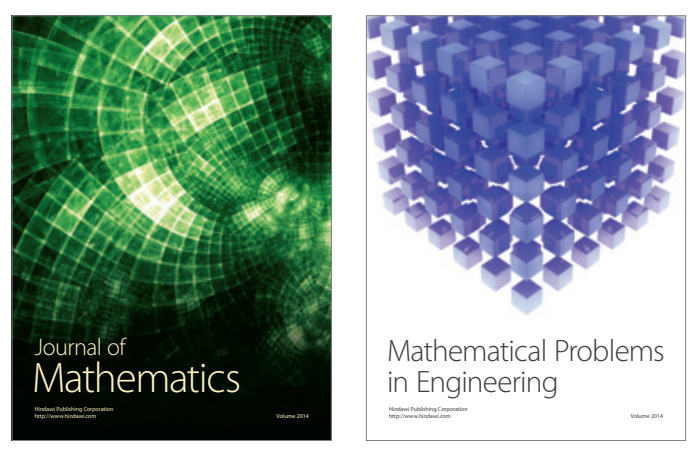

Mathematical Problems in Engineering
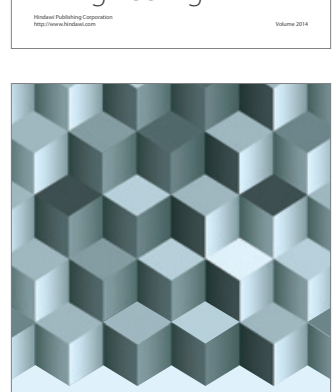

Journal of

Function Spaces
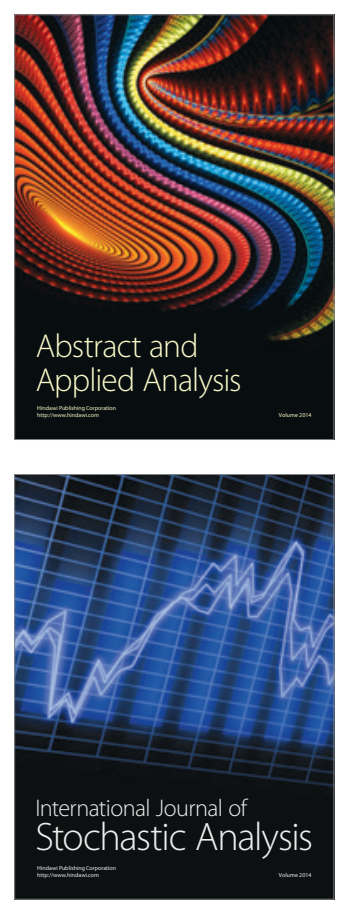

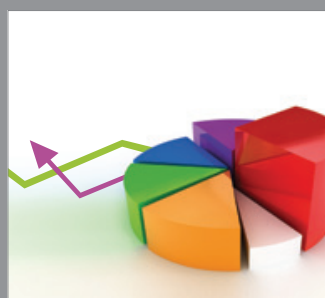

ournal of

Probability and Statistics

Promensencen
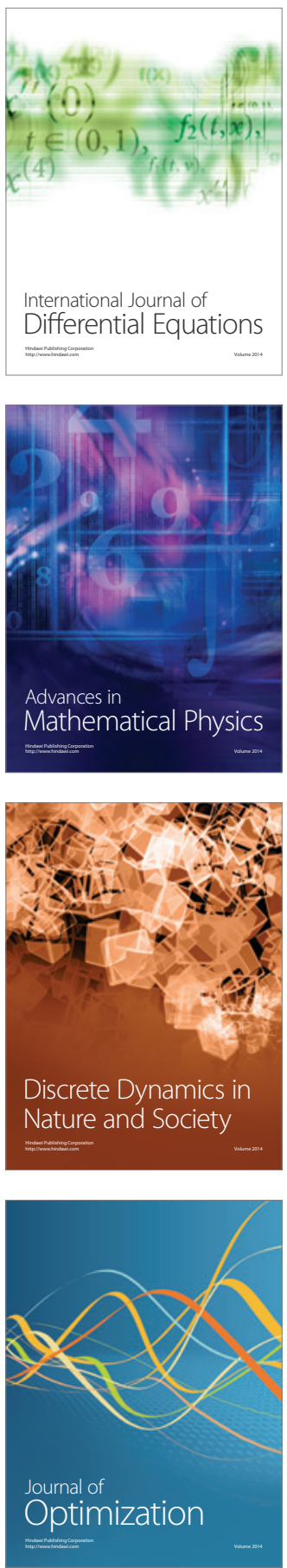